\title{
Towards realisation of an efficient continuous wave terahertz source using quantum dot devices
}

Nasir G. Bello, Semen Smirnov, Andrei Gorodetsky, Edik U. Rafailov

Nasir G. Bello, Semen Smirnov, Andrei Gorodetsky, Edik U. Rafailov, "Towards realisation of an efficient continuous wave terahertz source using quantum dot devices," Proc. SPIE 11124, Terahertz Emitters, Receivers, and Applications X, 111240G (6 September 2019); doi: 10.1117/12.2530730

SPIE Event: SPIE Optical Engineering + Applications, 2019, San Diego, California, United States 


\title{
Towards realisation of an efficient continuous wave Terahertz source using Quantum dot devices
}

\author{
Nasir G. Bello*a, Semen Smirnov ${ }^{\mathrm{a}}$, Andrei Gorodetsky ${ }^{\mathrm{b}}$, Edik U. Rafailov ${ }^{\mathrm{a}}$ \\ ${ }^{a}$ Aston Institute of Photonics Technologies, Aston University, Aston Triangle, Birmingham B4 7ET, UK \\ ${ }^{\mathrm{b}}$ Metamaterials Research group University of Birmingham B15 2TT, UK \\ ${ }^{b}$ ITMO University, St. Petersburg 197101, Russia.
}

\begin{abstract}
A continuous wave $(\mathrm{CW})$ terahertz source emitting in a broad frequency range $(1-5 \mathrm{THz})$ is promising towards achieving a compact, high power, finely tunable, room temperature terahertz generation system which will be of immense significance towards the realisation of terahertz applications in spectroscopy, communication, sensing, and imaging among others. We have demonstrated a tunable continuous-wave Quantum Dot external cavity laser emitting at two frequencies for continuous wave terahertz emission in a Quantum dot Photoconductive Antenna (PCA). The external cavity QD Laser has been characterised with tunability of $152 \mathrm{~nm}$ and a tuning range from $1143 \mathrm{~nm}-1295.8 \mathrm{~nm}$ that lies within the $\mathrm{THz}$ difference frequency for the generation of $\mathrm{THz}$ radiation from QD based PCAs.
\end{abstract}

Keywords: Terahertz emitters, Terahertz radiation, Quantum dots, Continuous wave operation, Photoconductive antennas

\section{INTRODUCTION}

A tunable compact continuous wave terahertz source is immensely desirable for various applications such as spectroscopy due to its high spectral resolution and for the realisation of compact and low-cost systems over pulsed terahertz system. The implementation of optical heterodyne down conversion in a photoconductive antenna and Terahertz quantum cascade lasers are the most widely used methods of generating continuous wave terahertz radiation. However, the required cryogenic cooling in THz quantum cascade lasers makes achieving a compactroom temperature terahertz source difficult to achieve. The use of dual wavelengths at a terahertz difference frequency in a Photoconductive antenna has been long used for generating continuous wave terahertz radiation [1-4]. The rise of semiconductor lasers and the appreciable properties of quantum dot gain media in semiconductor lasers has paved the way for widely tunable high power semiconductor lasers, moreover, the external cavity diode lasers provides narrow-linewidth that is suitable for $\mathrm{CW}$ terahertz emission. $\mathrm{CW} \mathrm{THz} \mathrm{signals} \mathrm{can} \mathrm{be} \mathrm{generated}$ using semiconductor materials with sub-picosecond carrier lifetimes optically pumped by a beat signal composed of two distinct narrow line width signals a few nanometres apart [5].

\subsection{Two-Colour Lasers for Continuous wave Terahertz}

Two-colour titanium-sapphire lasers have been explored due to their high spectral purity and tunability. Brown et al in 1993 incorporated 2 etalons in Ti:Sa lasers to realise a 2 colour laser [1]. However, Ti:Sa Lasers are expensive, complex and have a large footprint that makes them impractical for realisation of compact terahertz source [6]. Shortly after the first demonstration of $\mathrm{THz}$ photomixing; diode lasers have been discussed as an alternative pumping source for the photomixers [6]. However, they had power and linewidth limitations; however, the advancement in fabrication techniques has allowed for high power narrow linewidth diode lasers that are suitable for THz generation in PCAs. As demonstrated by E.U. Rafailov et al the use of QDs as the gain medium of the laser has enabled stable low threshold high power, stable laser system for THz generation [7]. Additionally the deployment of an External Cavity Diode Laser (ECDL) allows for wide tunability and narrow linewidth for tunable broad range $\mathrm{THz}$ emission.

Terahertz Emitters, Receivers, and Applications X, edited by Manijeh Razeghi,

Alexei N. Baranov, Miriam S. Vitiello, Proc. of SPIE Vol. 11124, 111240G

(C) 2019 SPIE · CCC code: 0277-786X/19/\$21 - doi: 10.1117/12.2530730 


\subsection{Quantum dot materials properties and growth}

Quantum dots are three dimensionally confined quantum structure that possess the ability to have tailored electronic structure with discrete energy levels and highly configurable properties which earned them the name "designer atoms"'[18]. They have been identified and investigated as potential candidates for lasers [19], detectors [20], single electron transistors, quantum computers [21] and for the interest of this research as PCA material for THz generation [17][22][4][23].

Achieving high quality dislocation-free Quantum dots has been primarily implemented by Molecular beam epitaxy which is a thin film epitaxial growth technique invented by Arthur Cho from Bell laboratories in 1968. Under high vacuum with different groups of atoms supplied a high thermal velocity molecular beam is formed which is made incident on a heated substrate surface to carry out MBE [22]. The QD laser and the QD material in the Photoconductive antenna for the purpose of this research was grown by MBE in the Strantski-Krastanow mode. Frank-van der Merwe Growth - The FM growth mode utilises a layer-by-layer growth mode routinely used for growth of Quantum well structures.

Strantski-Krastanow Growth is a hybrid of FM and Volmer weber growth modes. For moderate lattice mismatch, the growth is initiated layer by layer and the increase in the thickness of the epilayer accumulates strain due to lattice mismatch. At a critical thickness of the $2 \mathrm{D}$ layer, the strain is release to form small islands with the addition of epitaxial material the small islands will grow until desired [7].

\subsection{Quantum Dot Lasers}

The first realisation of semiconductor laser was in 1962 by groups in the United States and the then Soviet Union [8]. Dingle and Henry in 1976 proposed the idea of exploiting quantum effects in semiconductor lasers for achieving wavelength tunability and lower lasing threshold after making calculations that shows the advantages of using Quantum wells as the active layer in the lasers by capitalising on the change in density of states [9]. However, for over two decades lasers capitalising on structures with carrier confinement in two or three dimensions (i.e. quantum wire and quantum dot respectively) lacked practical realisation when compared to one dimensionally confined Quantum well lasers[10].

Over the years advances in epitaxial growth methods such as MBE has paved the way for sophisticated and highly controllable semiconductor lasers with many desirable properties as in Quantum dots [11]. The earliest demonstration of LDs that utilises a QD-based gain medium was in 1994 by Ledentsov et al [12] and Kirstaedter et al [13] with properties superior to quantum well lasers and reduced temperature sensitivity. A major design goal at the time was to improve the gain and lasing threshold alongside the reduction of the limiting thermal rollover effects reported in LDs by Ledentsov et al.[12].

Nader et al demonstrated a two-colour emission Quantum dot laser with an $8 \mathrm{THz}$ difference frequency as potential compact CW THz source [14]. Naftaly et al demonstrated a two-colour Ti:Sapphire laser with Fabry-perot etalon for $\mathrm{CW}$ terahertz generation and hence reducing the footprint of the $\mathrm{THz}$ source [15] thereby demonstrating that a quantum dot laser with smaller footprint than the Ti;sapphire enables a more compact $\mathrm{THz}$ source. A tunable terahertz beat signal from 1 to $2.21 \mathrm{THz}$ was demonstrated by Jiao et al from an InAs/InP Quantum dot modelocked laser combined with external cavity of two fibre Bragg gratings, the dual modes are phase correlated and the generated $\mathrm{THz}$ have less phase noise [16]. The design in this experiment owes to the work of Ksenia et al and Leyman et al where they demonstrated an external cavity Quantum dot laser for an ultra-compact, room temperature, tunable terahertz generation [4] [17]. Figure 1 shows a semiconductor laser utilising quantum dots in the gain media for high power, low threshold stable operation. A similar laser structure described in the exsperimental set-up section was used with two diffraction gratings to achieve stable two-colour operation for the generation of continuous wave $\mathrm{THz}$ radiation. 


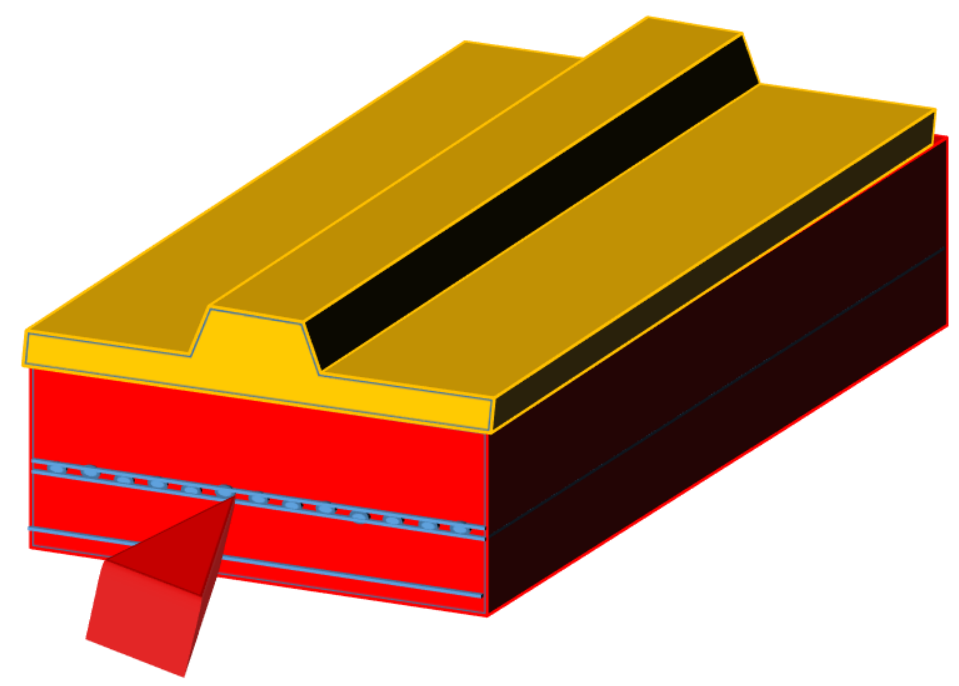

Figure 1. A schematic of a semiconductor laser utilizing Quantum dots as gain media.

\subsection{Quantum dots in PCAs}

The principle of operation of Photoconductive antennas relies on the generation of photocurrent and the movement of charge carriers. The THz signal generation layout in the experimental set-up as shown in figure 3; the two beams from the pump external cavity diode laser are focused on the gap of the photoconductive antenna placed between biased electrodes and is absorbed by the semiconductor, optical to terahertz conversion occurs from a generation of photocurrent that is modulated by the beat frequency from the two beams and radiated from the antenna as THz [17]. The quantum dos are integrated in the PCA for enhancement of the terahertz output as shown in the depiction in figure 2 .

E.U.Rafailov et al demonstrated an active layer of InAs quantum dot on GaAs, efficient emission of both pulsed and continuous wave THz signals with significant optical to $\mathrm{THz}$ conversion at $\leq 850 \mathrm{~nm}$ and $\leq 1300 \mathrm{~nm}$ [22]. The Quantum dot based THz PCA shown by Leyman et al and Ksenia et al was capable of being pumped at higher than $1 \mathrm{~W}$ optical mean power which is about 50 times higher than a conventional LT GaAs based PCA [4][24]. It also exhibits high carrier mobility and ultra-short photo carrier lifetime.

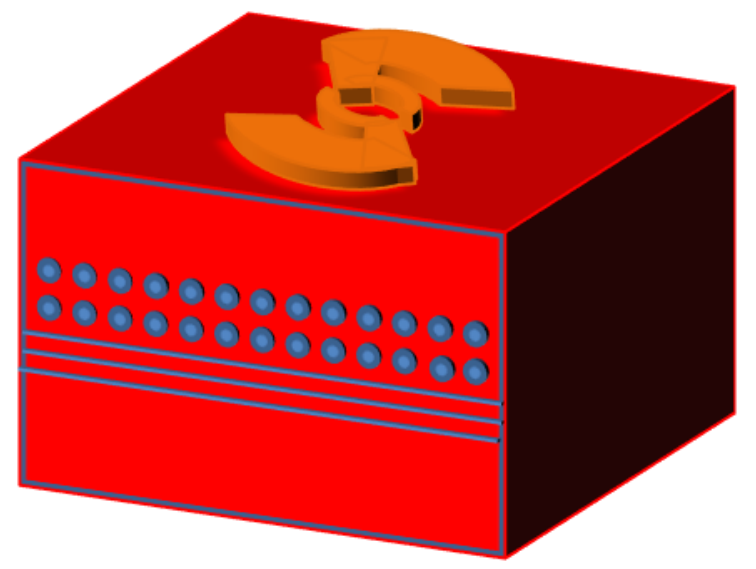

Figure 2. A depiction of a photoconductive antenna utilizing quantum dots for THz emission enhancement. 


\section{EXPERIMENTAL SETUP}

Figure 3 shows the layout of the system as implemented by [4], The QD structure used for the laser was grown on GaAs substrate and consists of 10 non-identical layers of InAs Quantum dots grown by molecular beam epitaxy in the Strantski-Krastanow mode The laser has a $5 \mu \mathrm{m}$ wide and $4 \mathrm{~mm}$ long waveguide angled at $5^{\circ}$ with respect to the normal of the back facet. Both laser facets have conventional anti-reflective (AR) coatings with total estimated reflectivity of $10^{-2}$ for the front facet and less than $10^{-5}$ for the angled facet.

The experimental set-up is based on an external cavity diode laser with simultaneous two-wavelength operation achieved using a beam splitter and two littrow gratings in each arm, wavelength scanning is achieved by rotating the diffraction gratings to be diffracted back along the incident beam. The laser was tested in single arm operation as shown in Figure 4 with a tunability of $152 \mathrm{~nm}$ achieved under an operation current of $1.7 \mathrm{~A}$ and temperature controlled at $20^{\circ}$ by a Thermoelectric cooler. Thereafter the laser was tested in the double grating quasi-littrow configuration, the tunability was made possibly across the $152 \mathrm{~nm}$ tunable bandwidth of the laser by changing the incidence angle of both gratings shown on figure 3.The optical beat of the two beams is incident on a photoconductive gap of a Photoconductive antenna and terahertz radiation is obtained from optical heterodyne down conversion (Photomixing).

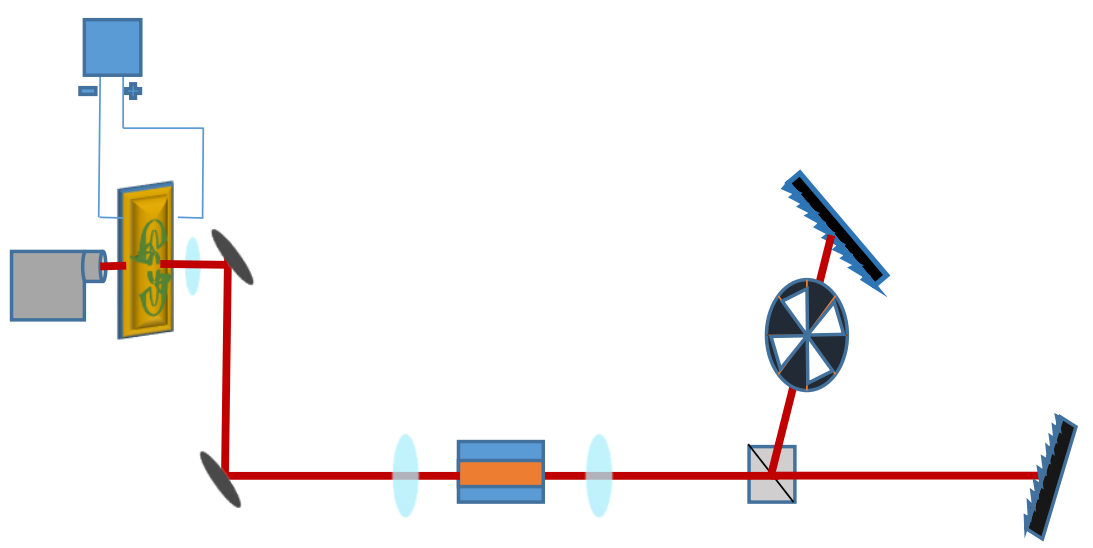

Figure 3. shows the set-up configuration for the generation of tunable continuous wave terahertz generation.

\section{RESULTS}

The graph below shows the tunability of the ECDL which lies within the THz frequency range for the generation of tunable continuous wave $\mathrm{THz}$ radiation. Stable dual-wavelength operation from the two laser arms was achieved across the range of $1143-1295 \mathrm{~nm}$ obtained wavelengths. The stable operation of the laser is significant for the efficient generation of terahertz radiation. The peak power of the laser is obtained at $1220 \mathrm{~nm}$ as $247 \mathrm{~mW}$. We have previously reported in the work of [4] et al $0.6 \mathrm{nW}$ at $0.83 \mathrm{THz}$ from $1157.4 \mathrm{~nm}$ and $1161 . \mathrm{nm}$ pump beams (mention antenna properties). However, the large photoconductive gap of $(50 \mu \mathrm{m}$ between the electrodes) was considered a hindrance towards generation of $\mathrm{THz}$ over $1 \mathrm{THz}$. The results from this work demonstrates the feasibility of a tunable compact room temperature Continuous wave terahertz source and emissions at over $1 \mathrm{THz}$ using smaller photoconductive gap. The Quantum dot PCA used by Ksenia et al was capable of being pumped at over $1 \mathrm{~W}$ which is up to 50 times more than the conventional low temperature grown GaAs. 


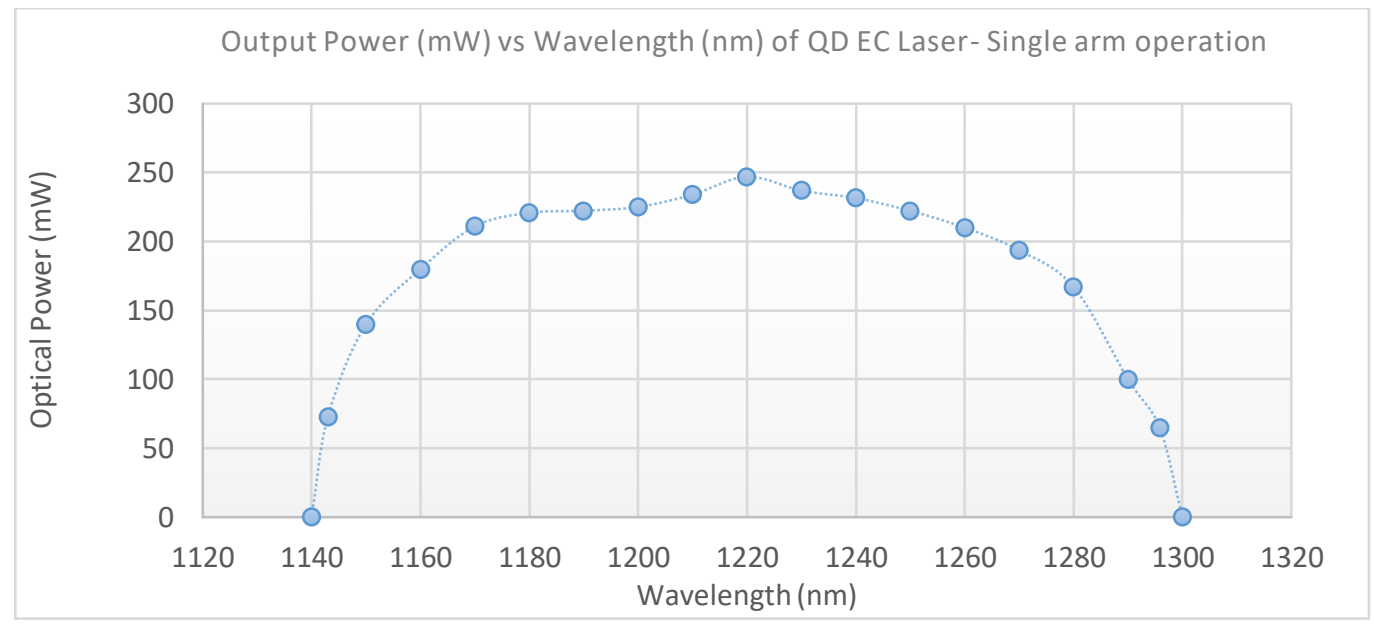

Figure 4: shows the tunability and output power operation of the laser in single arm operation.

\section{CONCLUSION}

The integration of quantum dot structures in lasers has enabled the development of lasers with the suitable requirements for the generation of continuous wave terahertz. We have demonstrated the appreciable properties of quantum dots towards realising a tunable, compact, room temperature continuous wave terahertz source. We have previously reported terahertz generation from quantum dot enhanced PCAs pumped by Quantum dot laser and propose the implementation of various quantum dot PCA design such as smaller photoconductive gap for the optimisation of the terahertz output.

\section{ACKNOWLEDGEMENT}

The Engineering and Physical Sciences Research Council (EPSRC) funded this work under grant EP/ R024898/1.

\section{REFERENCES}

[1] E. R. Brown, F. W. Smith, and K. A. McIntosh, "Coherent millimeter-wave generation by heterodyne conversion in low-temperature-grown GaAs photoconductors," J. Appl. Phys., vol. 73, no. 3, pp. 1480 1484, Feb. 1993.

[2] M. Tani, S. Matsuura, K. Sakai, and S. Nakashima, "Emission characteristics of photoconductive antennas based on low-temperature-grown GaAs and semi-insulating GaAs," Appl. Opt., vol. 36, no. 30, pp. 7853-7859, Oct. 1997.

[3] E. R. Brown, K. A. McIntosh, K. B. Nichols, and C. L. Dennis, "Photomixing up to $3.8 \mathrm{THz}$ in lowtemperature-grown GaAs," Appl. Phys. Lett., vol. 66, no. 3, pp. 285-287, Jan. 1995.

[4] K. A. Fedorova, A. Gorodetsky, E. U. Rafailov, and S. Member, "Compact All-Quantum-Dot-Based Tunable THz Laser Source,” IEEE J. Sel. Top. Quantum Electron., vol. 23, no. 4, 2017.

[5] T. Kruczek et al., Continuous wave terahertz radiation from an InAs/GaAs quantum-dot photomixer device, vol. 101. 2012.

[6] M. Tani, P. Gu, M. Hyodo, K. Sakai, and T. Hidaka, "Generation of coherent terahertz radiation by photomixing of dual-mode lasers," Opt. Quantum Electron., vol. 32, no. 4, pp. 503-520, 2000. 
[7] E. U. Rafailov, The Physics and Engineering of Compact Quantum Dot-based Lasers for Biophotonics. Wiley, 2014.

[8] R. N. Hall, G. E. Fenner, J. D. Kingsley, T. J. Soltys, and R. O. Carlson, "Coherent Light Emission From GaAs Junctions," Phys. Rev. Lett., vol. 9, no. 9, pp. 366-368, Nov. 1962.

[9] R. Dingle and C. H. Henry, "Quantum effects in heterostructure lasers," 1976.

[10] N. N. Ledentsov et al., "Quantum-dot heterostructure lasers," IEEE J. Sel. Top. Quantum Electron., vol. 6, no. 3, pp. 439-451, 2000.

[11] J. J. Coleman, "The development of the semiconductor laser diode after the first demonstration in 1962,” Semicond. Sci. Technol., vol. 27, no. 9, p. 90207, 2012.

[12] N. Ledentsov et al., Optical properties of heterostructures with InGaAs-GaAs quantum clusters, vol. 28. 1994.

[13] N. Kirstaedter et al., "Low threshold, large T/sub o/ injection laser emission from (InGa)As quantum dots," Electron. Lett., vol. 30, no. 17, pp. 1416-1417, 1994.

[14] N. A. Naderi, F. Grillot, K. Yang, J. B. Wright, A. Gin, and L. F. Lester, "Two-color multi-section quantum dot distributed feedback laser," Opt. Express, vol. 18, no. 26, pp. 27028-27035, Dec. 2010.

[15] M. Naftaly, M. R. Stone, A. Malcoci, R. E. Miles, and I. C. Mayorga, "Generation of CW terahertz radiation using two-colour laser with Fabry-Perot etalon ELECTRONICS LETTERS 3rd February 2005 ELECTRONICS LETTERS 3rd February 2005,” Electron. Lett., vol. 41, no. 3, 2005.

[16] Z. Jiao et al., "Tunable Terahertz Beat Signal Generation From an InAs / InP Quantum-Dot ModeLocked Laser Combined With External-Cavity,” vol. 24, no. 6, pp. 518-520, 2012.

[17] R. Leyman et al., "THz Emission from Quantum Dot-Based THz Antennas Pumped by a Tunable Quantum-Dot Laser Diode," in 2013 Conference on Lasers and Electro-Optics - International Quantum Electronics Conference, 2013, p. CC_P_5.

[18] M. A. Reed, "Quantum Dots," Sci. Am., vol. 268, no. 1, pp. 118-123, 1993.

[19] Y.-C. Xin et al., "InAs quantum-dot GaAs-based lasers grown on AlGaAsSb metamorphic buffers," J. Appl. Phys., vol. 94, no. 3, pp. 2133-2135, Jul. 2003.

[20] S. Raghavan et al., "Normal-incidence InAs/In0.15Ga0.85As quantum dots-in-a-well detector operating in the long-wave infrared atmospheric window $(8-12 \mu \mathrm{m})$," J. Appl. Phys., vol. 96, no. 2, pp. 10361039, Jun. 2004.

[21] A. Zrenner, E. Beham, S. Stufler, F. Findeis, M. Bichler, and G. Abstreiter, "Coherent properties of a two-level system based on a quantum-dot photodiode," Nature, vol. 418, p. 612, Aug. 2002.

[22] E. U. Rafailov, R. Leyman, D. Carnegie, and N. Bazieva, "Highly efficient quantum dot-based photoconductive THz materials and devices," 2013, vol. 8846, no., pp. 88460I-8846-10.

[23] H. Park, J. Kim, E. Jung, W. Choi, J. Lee, and H. Han, "Enhanced terahertz emission from InAs quantum dots on GaAs," 2006, vol. 6352, pp. 63521Z-6352-4.

[24] R. R. Leyman et al., "Quantum dot materials for terahertz generation applications," Laser Photon. Rev., vol. 10, no. 5, pp. 772-779, 2016. 